\title{
Importancia del control de la calidad de la baciloscopia en los laboratorios de diagnóstico de tuberculosis
}

\author{
Misleidis Sardiñas, Grechen García, María Rosarys Martínez, Raúl Díaz y Lilian M. Mederos
}

Instituto de Medicina Tropical
Pedro Kourí, La Habana, Cuba.
Laboratorio Nacional de Referencia
e Investigaciones de Tuberculosis,
Lepra y Micobacterias.
Financiamiento: Anual del
Ministerio de Salud Pública de
Cuba.
Conflictos de interés: No existe.
Recibido: 8 de enero de 2016
Aceptado: 26 de mayo de 2016

Correspondencia a: Misleidis Sardiñas Aragón misle@ipk.sld.cu

\section{Importance of quality control of baciloscopy in laboratories that perform diagnosis of tuberculosis}

Background: Baciloscopy is the primary tool for pulmonary tuberculosis diagnosis, being this technique the most used internationally in the search for infectious cases. Quality control is the process of the rechecking smears by a highly qualified observer. Aim: To evaluate and highlight the importance of quality control of smear microscopy in the Provincial Laboratories diagnosticians of Tuberculosis in Cuba. Methods: This study was conducted at the National Reference Laboratory and Research in Tuberculosis, Leprosy and Mycobacteria in the Institute of Tropical Medicine "Pedro Kouri", Havana, Cuba, Were evaluated 2676 smears received from January 2013 to December 2014, from Provincial Centers of Hygiene, Epidemiology and Microbiology of Cuba, including the special municipality Isla de la Juventud. Results: 2,664 (99.5\%) were concordant smears, the correlation obtained for the positive smears were $96.5 \%$ and $99.8 \%$ for negative. Were identified 12 reading errors: $7(3.5 \%)$ false positive and $5(0.2 \%)$ false negatives. Slides were classified with adequate quality of smears in $2039(76.2 \%)$, showed difficulties in realizing the extension in $1464(54.7 \%)$ and staining were adequate in 2343 (87.6\%). The kappa index was 0.9674. Conclusion: Although there was good agreement between observations it is recommended to improve the quality of extended, maintain staff training program that performs this activity, like regular supervision by specialists, to further improve the quality of diagnosis.

Key words: Baciloscopy, tuberculosis, quality control.

Palabras clave: Baciloscopia, tuberculosis, control de calidad.

\section{Introducción}

L a tuberculosis (TBC) es una enfermedad infecto-contagiosa causada por Mycobacterium tuberculosis. La Organización Mundial de la Salud reportó en el año 2014 que esta enfermedad afectó a 6,3 millones de personas en todo el mundo; de éstos $58 \%$ fue bacteriológicamente confirmado ${ }^{1}$.

La baciloscopia continúa siendo internacionalmente la herramienta primaria en el diagnóstico de la TBC pulmonar activa; esta es la prueba más utilizada no sólo en la búsqueda de casos infecciosos de la comunidad, sino además como medidor de la eficacia del tratamiento en estos pacientes ${ }^{2,3}$.

El control de calidad (CC) de la baciloscopia es un sistema diseñado para mejorar la habilidad, eficiencia y el uso de la microscopia, como opción de diagnóstico y monitoreo. Por otra parte, constituye un proceso de supervisión eficaz y sistemático de los resultados del trabajo de los laboratorios; asegura, además, que la información sea exacta, fiable y reproducible. Consta de tres componentes: CC (externo e interno), comprobación de habilidad y perfeccionamiento de la calidad del diagnóstico ${ }^{4-6}$. El $\mathrm{CC}$ externo es el proceso para determinar el nivel de desempeño del personal de laboratorio que incluye: la evaluación in situ, re-lectura de las láminas de esputo, el mejoramiento de la calidad, donde a través de los servicios de diagnóstico son analizadas las deficiencias encontradas y se toman medidas correctivas oportunas ${ }^{7,8}$.

En Cuba el Programa Nacional de Control de la Tuberculosis (PNCTB) y el Ministerio de Salud Pública trabajan conjuntamente con el objetivo de controlar y eliminar la TBC como un problema de salud. Dentro de las tareas para lograr este propósito se encuentra el mejoramiento del sistema de vigilancia, donde el CC de la baciloscopia (BK) es de suma importancia ${ }^{9,10}$.

El objetivo de nuestro trabajo fue evaluar y destacar la importancia del control de la calidad de la baciloscopia en los Laboratorios Provinciales encargados del diagnóstico de TBC en Cuba.

\section{Materiales y Métodos}

Se evaluó un total de 2.676 láminas de esputo, recibidas en el Laboratorio Nacional de Referencia e Investigaciones de Tuberculosis, Lepra y Micobacterias del Instituto de Medicina Tropical "Pedro Kourí" (LNRTBL-IPK), 
La Habana, Cuba, durante el período enero de 2013 a diciembre de 2014. Los Centros Provinciales de Higiene, Epidemiología y Microbiología (CPHEM), incluido el Municipio Especial Isla de la Juventud enviaron de forma mensual $10 \%$ de las láminas de esputo negativas, y 100\% de las láminas positivas.

La re-coloración y la codificación de las láminas se realizaron, según lo establecido en el $\mathrm{PNCTB}^{11}$, como se describe a continuación.

Para realizar la re-coloración de las láminas de esputo BAAR (bacilos ácido alcohol resistente) no fue necesario decolorar la lámina para realizar la nueva tinción y se filtró la fucsina antes de ser utilizada.

- Se cubrió con fucsina el portaobjeto y se aplicó calor hasta emisión de vapores, dejándose actuar por $10 \mathrm{~min}$.

- Se enjuagó con abundante agua y se escurrió.

- Se aplicó alcohol clorhídrico al 3\% por dos minutos.

- Se enjuagó con abundante agua y se escurrió.

- Se cubrió el portaobjeto con azul de metileno $1 \%$ por $45 \mathrm{seg}$.

- Se enjuagó con abundante agua y se dejó secar al aire.

Luego se procedió a realizar la codificación de la lámina de acuerdo al número de bacilos observados en cuatro líneas (observación aproximada 250-300 campos ópticos [CO]).

\begin{tabular}{|l|l|}
\hline $\mathbf{n}$ de bacilos & Codificación \\
\hline 0 en las 4 líneas & 0 \\
\hline 1 a 5 bacilos en las 4 líneas & El propio número \\
\hline 6 a 25 bacilos en las 4 líneas & 6 \\
\hline 25 o más bacilos en las 4 líneas & 7 \\
\hline 25 o más bacilos en 1 línea & 8 \\
\hline Bacilos en la mayoría de los campos & 9 \\
\hline
\end{tabular}

Para la evaluación de la calidad de las láminas enviadas se tuvo en cuenta:

\section{Calidad de las lecturas}

Se clasificaron los errores de la forma siguiente:

- Falso positivo (FP): Láminas consideradas positivas por el laboratorio evaluado, que resultaron negativas en el LNRTBL-IPK.

- Falso negativo (FN): Láminas consideradas negativas por el laboratorio evaluado, que resultaron positivas en el LNRTBL-IPK.

- Errores de codificación (EC): Láminas positivas, donde se obtuvieron diferencias cuantitativas en dos o más valores de codificación por parte de los evaluadores del LNRTBL-IPK, en relación a la codificación realizada por el laboratorio evaluado.
Los cálculos de las tasas de falsos positivos (FP) y falsos negativos (FN) se realizaron aplicando las siguientes fórmulas:

$$
\begin{aligned}
& \mathrm{FP}=\frac{\begin{array}{c}
\text { Láminas informadas positivas por el laboratorio } \\
\text { supervisado y negativas por el supervisor }
\end{array}}{\text { Total de láminas evaluadas }} \times 100 \\
& \mathrm{FN}=\frac{\text { Láminas informadas negativas por el laboratorio }}{\text { supervisado y positivas por el supervisor }} \\
& \text { Total de láminas evaluadas }
\end{aligned}
$$

Los valores adecuados de tolerancia de errores de lectura para un desempeño adecuado del laboratorio fueron para ${ }^{6,8-10}$ :

- FP: Hasta 5\%

- FN: Hasta $1 \%$

- EC: Hasta 5\%

\section{Calidad de la muestra}

Se evaluó de forma microscópica e indirecta (la calidad de la muestra) y se clasificó en:

- Mucopurulenta: Donde la mayoría de los campos presentaron leucocitos, además de mucus.

- Mucosa: Donde la mayoría de los campos presentaban mucus con muy aislados leucocitos.

- Saliva: Donde la mayoría de los campos se observaron células epiteliales, morfología bacteriana propia de microbiota normal y muy escaso mucus.

\section{Calidad del extendido}

Se evaluó macro y microscópicamente la extensión de la muestra y se clasificó en:

- Bueno: Si ocupó los 2/3 de la lámina portaobjeto, si el extendido estuvo homogéneamente distribuido y si la mayoría de los campos presentó cantidad suficiente de material, de manera que al mover el micrométrico se pudo observar de uno a tres niveles.

- Fino: La mayoría de los campos presentaron escaso material.

- Grueso: La mayoría de los campos presentaba abundante material y al mover el micrométrico se observó más de tres niveles.

- No homogéneo: El extendido presentó zonas finas y gruesas.

\section{Calidad de la coloración de Ziehl-Neelsen (ZN)}

- Buena: Láminas donde se pudo realizar la lectura de al menos 250 a $300 \mathrm{CO}$ sin cristales de fucsina y que no presentara falta de decoloración. 


\begin{tabular}{|c|c|c|}
\hline Indicadores & Parámetros que se miden & Valores esperados \\
\hline Calidad de las lecturas & Tasas de FP, FN y EC & $\begin{array}{l}\text { FP: Hasta } 5 \% \\
\text { FN: Hasta } 1 \% \\
\text { EC: Hasta } 5 \%\end{array}$ \\
\hline Calidad de la muestra & $\begin{array}{l}\text { Mucopurulenta } \\
\text { Mucosa } \\
\text { Saliva }\end{array}$ & $\begin{array}{l}\text { Hasta } 70 \% \text { de muestras mucosas o } \\
\text { mucopurulentas }\end{array}$ \\
\hline $\begin{array}{l}\text { Calidad de los } \\
\text { extendidos }\end{array}$ & $\begin{array}{l}\text { Bueno } \\
\text { Fino } \\
\text { Grueso } \\
\text { No homogéneo }\end{array}$ & Hasta $90 \%$ de extendido bueno \\
\hline $\begin{array}{l}\text { Calidad de la } \\
\text { coloración }\end{array}$ & $\begin{array}{l}\text { Buena } \\
\text { Buena (con cristales de fucsina) } \\
\text { Buena (con falta de decoloración) } \\
\text { Deficiente }\end{array}$ & $\begin{array}{l}\text { Hasta } 95 \% \text { de láminas bueno y/o } \\
\text { bueno con cristales de fucsina }\end{array}$ \\
\hline
\end{tabular}

- Buena (con cristales de fucsina): láminas donde se leyeron de 250 a $300 \mathrm{CO}$, a pesar de que se encontraron restos de cristales de fucsina básica fenicada.

- Buena (con falta de decoloración): láminas donde se observaron de 250 a $300 \mathrm{CO}$, a pesar de que en el resto del extendido presentaba decoloración insuficiente.

- Deficiente: la presencia de cristales o la falta de decoloración no permitieron leer correctamente al menos de 250 a 300 CO.

En la Tabla 1 se muestra un resumen de los indicadores evaluados, explicados anteriormente.

El análisis estadístico para determinar el índice de Kappa (IK) se realizó utilizando el Programa para Análisis Epidemiológico de Datos Tabulados EPIDAT, versión 3.1, con un intervalo de confianza del 95\%.

\section{Resultados}

Se evaluó un total de 2.676 láminas de esputos recibidas en el LNRTB-IPK. De éstas, 200 se recibieron como positivas y 2.476 como negativas. Se detectaron 12 errores de lectura; siete $(3,5 \%)$ falsos positivos, cinco $(0,2 \%)$ falsos negativos (Tabla 2$)$.

\begin{tabular}{|lccccc|}
\hline \multicolumn{2}{l}{ Tabla 2. Concordancia obtenida de acuerdo al total de láminas positivas y negativas } \\
\hline Láminas & Total de láminas & \multicolumn{2}{c}{ Concordantes } & \multicolumn{2}{c|}{ Discordantes } \\
& & No & $\%$ & No & $\%$ \\
\hline Positivas & 200 & 193 & 96,5 & 7 (FP) & 3,5 \\
Negativas & 2.476 & 2.471 & 99,8 & $5(\mathrm{FN})$ & 0,2 \\
\hline Total & 2.676 & 2.664 & 99,5 & 12 & 0,6 \\
\hline
\end{tabular}

El total de láminas concordantes fue de 2.664, para una concordancia general de 99,5\%. La concordancia obtenida para las láminas positivas fue de $96,5 \%$ y una discordancia de 3,5\%. Para las láminas negativas la concordancia encontrada fue de $99,8 \%$ con sólo $0,2 \%$ de discordancia, siendo estos valores aceptables para un desempeño adecuado del laboratorio (Tabla 2).

Los indicadores de calidad de forma general fueron aceptables. El estadígrafo IK fue de 0,9674 (intervalo de confianza $(0,9490-0,9858)$, siendo un valor muy cercano a 1 , para una concordancia elevada.

Con respecto a la calidad de la muestra, $2.039(76,2 \%)$ de las láminas evaluadas se calificaron como mucosas o muco-purulentas (calidad de la muestra adecuada) y $637(23,8 \%)$ muestras fueron saliva. Sobre la calidad del extendido se evaluaron con extendido adecuado $1.212(45,3 \%)$ láminas, y $1.464(54,7 \%)$ presentaron deficiencias en la realización de la extensión. La tinción fue buena o buena con cristales de fucsina en $2.43(87,6 \%)$ láminas. Se clasificó como tinción no adecuada 333 $(12,4 \%)$ láminas.

\section{Discusión}

El examen directo sigue siendo actualmente la técnica más apropiada para la detección de los pacientes con TBC pulmonar activa. El programa de control de calidad del examen directo de TBC debe estar adecuadamente implementado y monitoreado, lo cual es indispensable para obtener un elevado grado de confiabilidad. Su función primaria es identificar los laboratorios que necesiten un entrenamiento técnico adicional para realizar el examen microscópico, además de realizar adecuadas supervisiones periódicas con la finalidad de mejorar la calidad de los servicios de la microscopía ${ }^{11-13}$.

Durante la investigación se encontró una concordancia elevada $(99,5 \%)$, con una discordancia general de sólo $0,5 \%$. El IK muy cercano a $1(0,9674)$ obtenido en el estudio fue casi perfecto, según la clasificación de Viera, A. $\mathbf{J}^{14}$. Estos resultados fueron similares a los obtenidos por Martínez y cols., en un estudio realizado en los laboratorios de diagnóstico de TBC de los CPHEM de las provincias de Cuba en el año 2012 (Concordancia de $99,4 \%$ e IK $=0,8105)^{11}$.

La tasa de FP calculada en el estudio fue superior a la obtenida por Selvakumar y cols., en un trabajo realizado en la India durante el año 2003, donde la tasa de FP osciló de 0 a 1,2\%; por otro lado, la tasa de FN encontrada en la presente investigación fue menor $(0,2 \%)$ que la de este autor $(1,7-4,7 \%)$. Las diferencias en la identificación de FP pudieran estar dadas porque estos países poseen una alta carga de TBC, por lo que la positividad en los exámenes directos es mucho mayor; en consecuencia, 
existe un mayor número de láminas a evaluar de CC con una probabilidad mayor de encontrar más errores de lectura ${ }^{15-17}$.

Los cuatro errores de codificación (EC) que se identificaron en este estudio fueron en laboratorios diferentes. Según la literatura internacional ${ }^{12}$ se considera al EC un error de lectura si existe tendencia a repetir este parámetro en un mismo laboratorio, lo cual no sucedió en este trabajo.

La proporción de láminas con deficiencias en la extensión fue mayor de $50 \%$, superior a lo encontrado por Martínez y cols, en Cuba, en un estudio similar en el año 2006, donde obtuvo el porcentaje 5,8\% de láminas deficientes $^{6}$. Kuzsnier y cols en Santa Fé, Argentina, en el 2001, reportaron resultados inferiores a nuestra investigación con $34,7 \%$ de láminas donde se identificaron deficiencias técnicas relacionadas con la extensión ${ }^{15}$. Las deficiencias obtenidas en el presente estudio pudieran estar relacionadas por la poca estabilidad del personal de laboratorio dedicado a realizar esta actividad durante el período de estudio y la incorporación de técnicos sin la experiencia necesaria. Para mejorar la calidad del diagnóstico de la TBC es imprescindible que el personal este previamente entrenado en realizar el examen directo.

El control de calidad de la muestra, desde el punto de vista del procedimiento microscópico, hasta este momento no se realizaba como parte del CC de la BK en Cuba. Por primera vez, éste se introduce en el PNCTB, lo que ha permitido determinar de forma indirecta la calidad de las mismas. Este estudio determinó que la proporción de muestras mucosas y mucopurulentas fue de $76,2 \%$, valores mayores de lo esperado. Ésta resulta similar a la descrito por Kuzsnier y cols., en el año $2001(73,7 \%)^{15}$.

El control de la calidad del extendido y la coloración de $\mathrm{ZN}$ arrojó valores por debajo de lo esperado según indica la literatura internacional. Al realizar la coloración de $\mathrm{ZN}$, es sumamente importante utilizar una adecuada técnica, pues ésta puede influir en gran medida en la calidad de la lectura y ser causa de resultados FP y FN. Aproximadamente $46 \%$ de los FP han sido relacionados con deficiencias en la coloración, tales como: la presencia de cristales de fucsina que, en ocasiones puede traer confusión al microscopista, si éste no tiene suficiente experiencia para diferenciarlos de los bacilos, y la pobre decoloración con el alcohol ácido. Por otra parte, un insuficiente calentamiento durante la tinción, puede afectar el grado de retención de la fucsina por el bacilo y producir resultados falsamente negativos durante la realización de la relectura de las láminas. Otra causa de FN sería la poca concentración bacilar por $\mathrm{mL}$ de muestra ${ }^{15,17}$.

Existen otras causas que pueden influir en las diferencias entre las lecturas. Los BAAR pueden volverse invisibles después de dos a tres semanas bajo condiciones de alta temperatura, humedad y exposición a luz solar. Por otro lado, la fucsina básica no es estable en altas temperaturas y climas húmedos, lo que ocasionaría $\mathrm{FN}$; de ahí que la incorporación de la re-coloración de las láminas antes de realizar la relectura, es de gran importancia para evitar errores ${ }^{12,17-20}$.

De forma general, podemos decir que aunque hubo una concordancia adecuada entre el LNRTBL-IPK y los laboratorios provinciales encargados del diagnóstico de TBC, se identificaron deficiencias al realizar el extendido y en la coloración de ZN. Como consecuencia, recomendamos no descuidar las continuas supervisiones, mantener un programa de entrenamiento constante del personal que realiza esta actividad, para continuar mejorando la calidad de la baciloscopia o examen directo BAAR, herramienta extremadamente útil en los laboratorios de la red de Cuba.

\section{Resumen}

Introducción: La baciloscopia es la herramienta primaria en el diagnóstico de la tuberculosis (TBC) pulmonar activa, siendo esta la técnica más utilizada internacionalmente en la búsqueda de casos infecciosos. El control de calidad consiste en la relectura de las láminas por un observador altamente calificado. Objetivo: Evaluar y destacar la importancia del control de la calidad de la baciloscopia en los laboratorios provinciales encargados del diagnóstico de TBC en Cuba. Material y Métodos: Este estudio fue realizado en el Laboratorio Nacional de Referencia e Investigaciones de Tuberculosis, Lepra y Micobacterias del Instituto de Medicina Tropical "Pedro Kourí”, La Habana, Cuba. Fueron evaluadas 2.676 láminas recibidas en el período de enero de 2013-diciembre de 2014, procedentes de los diferentes Centros Provinciales de Higiene, Epidemiología y Microbiología de Cuba, incluido el Municipio Especial Isla de la Juventud. Resultados: Hubo 2.664 (99,5\%) láminas concordantes, la concordancia obtenida para las láminas positivas fue $96,5 \%$ y las negativas $99,8 \%$. Se identificaron 12 errores de lectura: $7(3,5 \%)$ falsos positivos, $5(0,2 \%)$ falsos negativos. Se calificaron láminas con calidad de la muestra adecuada en $2.039(76,2 \%)$, presentaron deficiencias en la realización de la extensión 1.464 (54,7\%), y la tinción fue adecuada en $2.343(87,6 \%)$. El índice de kappa fue de 0.9674. Conclusión: Aunque hubo una adecuada concordancia entre las observaciones realizadas, se recomienda mejorar la calidad del extendido, mantener programa de entrenamiento al personal que realiza esta actividad, al igual que las supervisiones periódicas por parte de especialistas, para continuar mejorando la calidad del diagnóstico. 


\section{Referencias bibliográficas}

1.- WHO Report. Global Tuberculosis Report. 20th Edition WHO Library Cataloguing-inPublication Data, 2015.

2.- Minion J, Zwerling A, Pai M. Diagnostics for tuberculosis: what knowledge did we gain through the International Journal of Tuberculosis and Lung disease in 2008. Int J Tuberc Lung Dis 2009; 13 (6): 691-7.

3.- Steingart K R, Ng V, Henry M. Sputum processing methods to improve the sensitivity of smear microscopy for tuberculosis: a systematic review. Lancet Infect Dis 2006; 6 (10): 664-74.

4.- Manual para el diagnóstico bacteriológico de la tuberculosis. Normas y guía técnica, parte I Baciloscopia. OMS. 2008.

5.- Rieder H L, Chonde T M, Myking H, Urbanczik R, Kim S I, Laszlo A, et al. The Public Health Service National Tuberculosis Reference Laboratory and the National Laboratory Network. France: IUATLD; 1998.

6.- Martínez Romero M R, Sardiña Aragón M, García León G, Díaz Almaguer M, Llanes Cordero M J, Montoro Cardoso E. Evaluación del control de calidad de la baciloscopia en el diagnóstico de la tuberculosis en Cuba. Rev Cubana Med Trop 2006; 58 (3): 42-5.

7.- Rieder H L, Chonde T M, Myking H, Urbanczik R, Kim S I, Laszlo A, et al. The Public Health Service National Tuberculosis
Reference Laboratory and the National Laboratory Network. France: IUATLD; 1998.

8.- Aziz M A, Ba F, Becx-Bleumink M, Britzel G, Humes R, Lademarco M F, et al. External quality assessment for AFB smears microscopy. Washington, DC: Microscopy Association of Public Health Laboratories, 2002.

9.- González E, Armas L, Llanes M J. Progress towards tuberculosis elimination in Cuba. Int $\mathrm{J}$ Tuberc Lung Dis 2007; 11 (4): 405-11.

10.- Martínez Romero M R, García León G, Sardiña Aragón M, Montoro Cardoso E. Control de calidad de la baciloscopía de esputo BAAR en laboratorios provinciales en Cuba. Rev Cubana Hig y Epidemiol 2012; 50 (1): 29-36.

11.- Programa Nacional de Control de la Tuberculosis. Manual de Normas y Procedimientos. La Habana: Ed. Ciencias Médicas; 2013.

12.- Instituto Nacional de Enfermedades Respiratorias "Dr. Emilio Coni. Instituto Nacional de Enfermedades Infecciosas. ANLIS “Carlos G. Malbrán, Red Nacional de Laboratorios de Tuberculosis. Argentina. Microscopia. Argentina: Normas Técnicas; 2000.

13.- Enfermedades Infecciosas. Tuberculosis. Diagnóstico de Tuberculosis. Guía para el equipo de Salud. Editado por Dirección de Epidemiología, Ministerio de Salud y
Presidencia de la Nación, República de Buenos Aires, Argentina, 2009.

14.- Viera A J, Garrett J M. Understanding interobserver agreement: the kappa statistic. Fam Med 2005; 37 (5): 360-3.

15.- Kusznier G F, Latini O A, Sequeiro M D. Quality assessment of smears microscopy for acid fast bacilli in the Argentine Tuberculosis Laboratory Network, 1983-2001. Int J Tuberc Lung Dis 2004; 8 (10): 1234-41.

16.- Fujiky A, Giango C, Endo S. Quality control of sputum smears examination in Cebu province. Int J Tuberc Lung Dis 2002; 6 (1): 39-46.

17.- Selvakumar N, Sivagamasundari S, Prabhakaran E, Govindaraju R, Perumal M, Granich R, et al. Storage of heat-fixed unstained sputum AFB smears for panel testing in a tuberculosis unit in South India. Int J Tuberc Lung Dis 2005; $\mathrm{m} 9$ (2): $\mathrm{m} 223-5$.

18.- Rohit S, Neeta S, Mukerjee S, Sharma P P. RNTCP: Quality control of sputum microscopy at sub-district level. Ind J Tub 2002; m49: m143-5.

19.- Buzingo T, Sanders M, Masabo J P, Nyandwi S, Van Deun A. Systematic restaining of sputum smears for quality control is useful in Burundi. Int J Tuberc Lung Dis 2003; 7 (5): 439-44.

20.- Tuberculosis. Detección de casos, tratamiento y vigilancia. Preguntas y respuestas. Kurt Toman. Segunda edición. Ed. Thomas R. Frieden. Publicación Científica y Técnica No 617. OMS 2006. 